Musées, Patrimoine et Culture scientifiques et techniques

$164 \mid 2016$

mars-avril 2016

\title{
Patrimoine des universités et médiation culturelle des sciences
}

Sébastien Soubiran

(2) OpenEdition

Journals

Édition électronique

URL : http://journals.openedition.org/ocim/1649

DOI : 10.4000/ocim. 1649

ISSN : 2108-646X

Éditeur

OCIM

Édition imprimée

Date de publication : 1 mars 2016

Pagination : 33-37

ISSN : 0994-1908

Référence électronique

Sébastien Soubiran « Patrimoine des universités et médiation culturelle des sciences », La Lettre de I'OCIM [En ligne], 164 | 2016, mis en ligne le 01 mars 2017, consulté le 21 avril 2019. URL : http:// journals.openedition.org/ocim/1649; DOI : 10.4000/ocim.1649

Ce document a été généré automatiquement le 21 avril 2019

Tous droits réservés 


\title{
Patrimoine des universités et médiation culturelle des sciences
}

\author{
Sébastien Soubiran
}

L'article (disponible sur www.ocim.fr, rubrique La Lettre de l'OCIM) détaillait notamment les objectifs de la démarche, le contexte dans lequel elle s'inscrivait et les partenaires mobilisés.

\section{Patrimoine des universités} et médiation scientifique
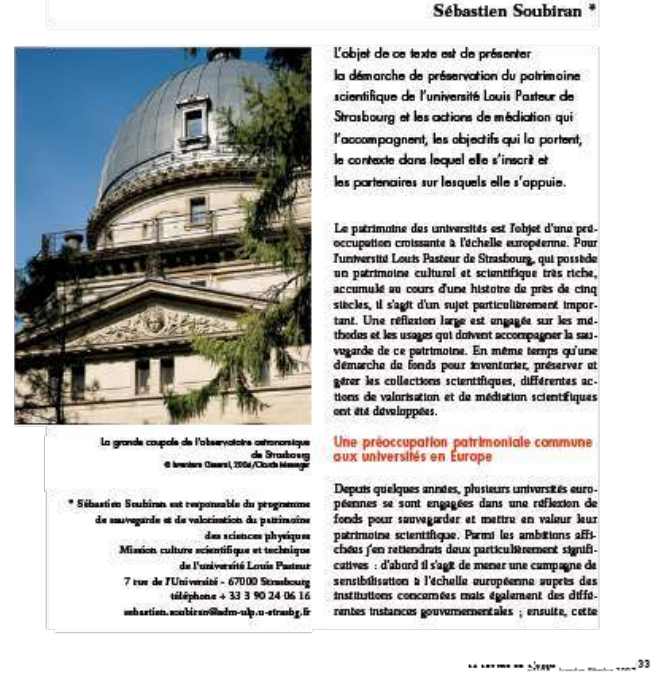

1 L'exercice, initié l'an dernier pour les trente ans de l'OciM, de revisiter un article quelques années plus tard, est particulièrement enrichissant pour suivre l'évolution d'une pratique professionnelle et son environnement. Ma contribution publiée en 2007 rendait compte des actions de médiation scientifique engagées à l'université de Strasbourg 
mobilisant le patrimoine des universités. Ces activités étaient présentées en regard d'un contexte local, national et européen qui poussait, à investir le champ patrimonial dans les universités pour développer une politique de mise en public des savoirs.

Presque dix années se sont écoulées et force est de constater que des changements se sont opérés tant du point de vue institutionnel, que des pratiques professionnelles, des communautés impliquées ou des enjeux à l'œuvre. Bien que les situations restent fortement contrastées, à Strasbourg, comme en France ou en Europe, le patrimoine des universités, musées et collections semblent avoir trouver différents chemins pour accompagner les universités dans leurs missions fondamentales au gré des mutations engagées et des changements observés dans le champ de la médiation culturelle des sciences. Sans reprendre point par point ma contribution de 2007, je propose, dans un premier temps, de relever les changements les plus marquants, puis d'en présenter les impacts sur la préservation du patrimoine des universités et son rôle dans la médiation culturelle des sciences. Partant du contexte européen, puis national, je terminerai par le cas local de l'université de Strasbourg.

\section{Une mutation profonde des universités}

3 Partout en Europe, les universités vivent aujourd'hui une période charnière et sont amenées à repenser leur rôle dans le développement de la connaissance. Depuis le processus de Bologne en 1999, qui a conduit à la mise en place d'un espace européen de l'enseignement supérieur en 2010, ou la stratégie de Lisbonne, qui pose les bases d'une nouvelle interconnexion entre économie et université "l'économie de la connaissance". Cette vision libérale portée par l'OCDE (Organisation de coopération et de développement économiques), la banque mondiale ou l'OMC (Organisation mondiale du commerce), impacte les modalités de financement des universités et induit un mode de gouvernance plus entrepreneurial (mise en compétition des universités, laboratoires, existence de classements, incitation à la diversité des financements, rôle accru des régions) ${ }^{1}$. En France, ce processus se traduit concrètement en 2007 avec la loi relative aux libertés et responsabilités des universités, dite loi LRU. Elle s'accompagne d'un processus de changement d'échelle et de mutualisation de moyens pour les universités avec la mise en place des PRES (Pôles de recherche et d'enseignement supérieur), puis des COMUEs (Comunautés d'universités et d'établissements) avec pour objectif la fusion de différents établissements. Ce management concurrentiel pousse à la construction ou la réaffirmation d'une "identité" forte de chaque université. Celle-ci se traduit fréquemment par la volonté chez les équipes dirigeantes de développer ou renforcer l'ouverture de l'université sur la Cité et par une attention, sinon nouvelle du moins accrue, envers les processus de construction identitaire et leurs instruments potentiels. Dans ces nouvelles universités on a vu émerger des chargés de mission voire des vice-présidence "science et société" qui témoignent de cette volonté d'ouverture et de réfléchir à la mise en œuvre de sa mission de diffusion de la connaissance. La culture, l'histoire, le patrimoine, que ce soit pour les nouvelles universités ou les COMUEs sont dans certains cas pensés comme des outils à même d'aider à la construction ou la recomposition d'une identité et offrent un moyen de s'inscrire de fait dans le territoire qui partage cette histoire, ce patrimoine, cette culture. 


\section{Les collections scientifiques comme instruments de recherche}

Outre les dimensions culturelle et sociale des collections, les valeurs pédagogique et scientifique des collections universitaires, trouvent un écho parmi certaines institutions en charge de la gouvernance de la recherche et/ou les universités.

5 En janvier 2011, la Wissenschaftsrat, conseil national scientifique allemand, a publié une liste de recommandations dans laquelle il souligne le fort potentiel de recherches porté par ces collections. Ces recommandations ont permis le financement par le ministère fédéral de l'Éducation et de la Recherche d'un centre de coordination pour les collections scientifiques. Situé au Hermann von Helmholtz-Zentrum für Kulturtechnik de l'université Humboldt de Berlin il mène une action à l'échelle nationale (http://wissenschaftlichesammlungen.de/en/). En écho à cette initiative, le gouvernement portugais avec les instances européennes de la recherche a intégré les collections scientifiques dans son programme national de grands équipements de recherche. PRISC (Portuguese Infracstructures of scientific collections) est coordonné par le musée d'histoire des sciences de l'université de Lisbonne, en collaboration avec ses homologues de Coimbra et Porto. En France, e-ReColNat coordonné par le Muséum national d'Histoire naturelle (MNHN) porte un programme de numérisation des collections d'Histoire naturelle: herbier, paléontologie et zoologie. Même si la gouvernance, les enjeux et les communautés impliqués sont différents d'un pays à l'autre, l'émergence de grandes infrastructures de recherche basées sur les collections scientifiques marque très clairement une prise de conscience du potentiel scientifique, pluridisciplinaire, des collections universitaires.

\section{Renforcement d'un travail en réseaux en Europe}

6 À l'échelle européenne une plus grande structuration et action en réseau a été portée par le réseau Universeum, conjointement avec UMAC (University Museum and Academic Collections, comité thématique international de l'ICOM, créé en 2001). Universeum s'est constitué en association en 2011 avec l'objectif de pérenniser et renforcer les actions portées par ce réseau créé en 2000. Aujourd'hui, ce réseau comporte une centaine de membres individuels et une vingtaine de membres institutionnels répartis dans 24 pays européens (universeum.it). Le nombre croissant de participants aux conférences annuelles et la diversité des présentations proposées rendent compte de la richesse en termes de recherches et d'actions autour du patrimoine des universités. Les interventions témoignent également de situations encore très disparates d'un pays à l'autre et dans les universités d'un même pays.

7 L'une des fragilités tient au fait que les moyens investis pour la préservation et la valorisation du patrimoine reposent souvent sur des volontés et des engagements individuels et s'appuient rarement sur un personnel et des structures pérennes. De fait, les outils pour mener une action de lobbying efficace sont encore à construire. On manque en effet d'une réelle connaissance/vision d'ensemble des publics touchés, d'une cartographie précise des acteurs engagés dans la préservation du patrimoine des universités, des différentes politiques en place, modèles économiques ou best practices. 
L'émergence de nouveaux réseaux nationaux, portée par des initiatives nationales de grande ampleur, participe toutefois à la construction d'outils communs, de bases de données, de publications, colloques et actions politiques communes.

\section{Une réaffirmation d'un dialogue nécessaire entre science et société}

Les défis et débats générés par l'essor des sciences et des techniques dans les sociétés contemporaines sont plus que jamais au cœur de multiples réflexions et interrogations. Pourtant, malgré les actions déployées depuis de nombreuses années, tout porte à croire que des solutions satisfaisantes ne sont toujours pas apportées. Parmi les questions les plus discutées figurent celle de la mise en culture des sciences et des techniques, celle de la socialisation des savoirs scientifiques ou encore celle des modalités d'organisation de débats publics et de choix démocratiques relatifs aux sciences et techniques. En France, plusieurs rapports d'expertise convergent sur la nécessité de repenser un nouveau pacte entre sciences et sociétés, on parle alors de sciences citoyennes, participatives, de recherche et innovation responsable ${ }^{2}$. Ce constat s'accompagne d'une mutation profonde des pratiques de médiation développées par les acteurs de terrain qui s'appuient d'une part sur les nouveaux outils du numérique et d'autre part sur la stimulation et la valorisation de la pratique amateur dans le champ des sciences et des techniques, ou la création collaborative: conventions de citoyens, recherche participative, living lab, expertise collective mixte. En France, les projets financés dans le cadre des investissements d'avenir pour la culture scientifique et technique à partir de 2011 rendent bien compte de ces changements à l'œuvre, si l'on pense par exemple aux nouveaux espaces et outils créés par les partenaires du programme Inmédiats.

Quelle rôle les universités jouent-elle dans l'accompagnement de ces changements et existe-t-il une place pour le patrimoine des universités dans ce nouvel écosystème?

\section{En France un paysage contrasté et une action en réseau difficile}

10 En France, le premier rapport sur les universités, publié en 2011 par l'Observatoire du patrimoine et de la culture scientifiques et techniques de l'OCIM, fait état d'une grande variété d'actions ainsi que d'une grande richesse patrimoniale. Cette première enquête met également en avant un manque de stratégie d'ensemble entre patrimoine et actions de CSTI et montre l'existence d'actions peu visibles avec (malgré quelques exceptions) un impact médiocre à l'échelle locale ou nationale au regard de la richesse des ressources humaines et patrimoniales. Une corrélation peut être faite avec le cloisonnement important entre les services universitaires en charge de la CST (plus centralisé) et ceux en charge des collections (plus souvent liés à des composantes) mais aussi du fait d'actions en CST s'inspirant davantage d'activités de médiation des centres de sciences ou des CCSTI et donc peu basées sur des collections scientifiques.

11 Malgré une présence renforcée dans les réseaux professionnels de CSTI ou des musées à l'échelle nationale et internationale ${ }^{3}$, les universités doivent encore trouver leur place et leur rôle dans la médiation des sciences et des techniques en complémentarité des CCSTI, des musées et associations d'éducation populaire. Le paysage encore changeant des 
universités et des COMUEs rend difficile la stabilisation et donc la construction d'une action collective ou d'un réseau. Plusieurs initiatives engagées par des réseaux nationaux tels que l'AMCSTI ou Art+Université+Culture, ou encore entre les acteurs de la CST dans les universités, montrent toutefois la volonté et la nécessité de formaliser l'identification de notre communauté, comme ont pu le faire par exemple les muséums en créant la Conférence permanente des muséums de France.

\section{Naissance du Jardin des sciences de l'université de Strasbourg}

Le $1^{\text {er }}$ janvier 2009, les trois universités strasbourgeoises furent les premières en France à opérer une fusion. Une vice-présidence science et société est créée confirmant l'importance accordée par le nouveau président Alain Béretz d'ouvrir l'université vers la cité. Dans la dynamique de cette fusion, le planétarium et la mission culture scientifique et technique sont réunis dans un même service, le Jardin des sciences. Ce rapprochement donne naissance à une structure culturelle hybride à même de construire des actions de médiation scientifique, déployée dans l'ensemble du territoire, mobilisant différents registres et outils pour un public varié. L'étude, la préservation et la mise en public des collections et du patrimoine dans son ensemble enrichissent les activités développées et en retour bénéficient d'une attention et d'une visibilité accrue au sein des composantes et personnels de l'université mais également auprès des partenaires culturels locaux. Je développerai trois évolutions qui sont à mon sens marquantes et significatives depuis 2007 dans la façon dont les collections et structures muséales participent à l'ouverture de l'université de Strasbourg sur la cité, au dialogue science et société et au développement d'une politique de médiation culturelle et sociale des sciences.

\section{Un patrimoine au cœur du paysage culturel et muséal de la ville et de sa région}

L'opération d'inventaire du patrimoine scientifique de l'université engagé en partenariat étroit avec le service de l'Inventaire du patrimoine de la Région Alsace, démarré autour du patrimoine de la physique, s'est déployée sur l'ensemble du campus historique. L'approche globale de la méthode d'inventaire adoptée pour chaque bâtiment a ainsi permis de mettre en avant la cohérence d'ensemble qui portait le projet politique, scientifique et architectural de la Kaiser Wilhem Universität construite par les allemands à partir de 1870. L'université, au cœur du projet urbain de la nouvelle ville allemande - la Neustadt, a inauguré en 2012 la campagne d'inventaire menée par la Région Alsace et la Ville de Strasbourg sur ce quartier, ainsi que les Rendez-vous de la Neustadt, événement annuel conçu pour restituer au grand public les avancées de l'enquête. Ces collaborations étroites avec les services du patrimoine de la Ville de Strasbourg et de la Région Alsace ont ainsi renforcé d'une part la visibilité de l'université et de son patrimoine dans le paysage culturel local et régional et d'autre part l'accessibilité de patrimoine en développant de nouveaux outils accessibles à un large public. Chaque année ce sont plus de 15000 personnes qui visitent les collections et le patrimoine des universités lors des événements tels que la Nuit des musées et les Journées européennes du patrimoine. 
14 Un autre partenariat, engagé avec la direction des musées de la Ville de Strasbourg, a renforcé de façon significative la visibilité et l'accessibilité du patrimoine universitaire. Cette collaboration s'appuie notamment sur la double tutelle du musée zoologique partagée entre l'université et la ville depuis le début du XIX ${ }^{\mathbf{e}}$ siècle. La direction des musées a également associé l'université dans la programmation de la deuxième tranche du musée historique de la Ville de Strasbourg afin de souligner le rôle marquant de l'université. Plusieurs objets des collections de l'université ont été mises en dépôt au musée historique et sont exposés dans l'exposition permanente pour la partie 1800-1948. Le partenariat avec l'université et le Jardin des sciences est également sollicité dans le cadre d'expositions temporaires. L'exposition L'Europe des Esprits ou la fascination pour l'occulte: 1750-1950, installé en 2011 au musée d'Art moderne et contemporain de la Ville de Strasbourg sur plus de $2500 \mathrm{~m}^{2}$, traitait de la question de l'occulte et du spiritisme dans l'art, la littérature et la science. La prochaine grande exposition des musées pour l'automne 2017, Strasbourg, laboratoire d'Europe, 1880-1930, explorera l'histoire culturelle, artistique et intellectuelle de Strasbourg. Ces collaborations permettent de démontrer dans des dimensions tout à fait exceptionnelles la valeur intrinsèque des collections universitaires et la dimension culturelle des savoirs scientifiques.

\section{Le patrimoine comme outil de médiation culturelle et sociale des sciences}

15 L'action scolaire et éducative du Jardin des sciences s'appuie sur les ressources spécifiques de l'université de Strasbourg, en premier lieu les acteurs de la recherche scientifique, mais aussi les collections et structures muséales et sur le planétarium fixe et mobile. Ces actions ont lieu à la fois au sein de l'université mais également hors les murs (cafés scientifiques, malles pédagogiques, expositions, planétarium mobile par exemple). Elles s'appuient également sur une collaboration étroite avec le rectorat. Toutes nos actions scolaires sont donc co-construites par des chercheurs/enseignants-chercheurs, professeurs relais du rectorat, et les médiateurs du Jardin des sciences. À ce trinôme s'ajoutent les chargés de collections.

16 En complément de ces actions éducatives, le soutien financier de l'Initiative d'excellence (IdEx) du programme Investissements d'avenir, dont l'université de Strasbourg est lauréate, nous a permis de nous engager dans de nouveaux projets. Certains d'entre eux mobilisent le patrimoine de l'université pour construire une médiation culturelle et sociale des sciences qui s'inscrit à la fois dans notre volonté de participer au renouveau du débat public sur les sciences et de démontrer la pertinence de recourir à l'histoire, aux sciences humaines et sociales et au patrimoine pour le faire. Deux expositions seront ainsi inaugurées en avril et mai 2016. Lumière! Explorer l'impossible co-produite avec le musée zoologique et Sismo Strasbourg co-produite avec l'École et Observatoire des sciences de la Terre (EOST), mobilisent l'histoire, le patrimoine et les collections scientifiques. En les mettant en dialogue avec les connaissances et enjeux sociétaux actuels, le visiteur est placé au cœur des processus et des pratiques qui accompagnent la construction d'une connaissance scientifique afin de mieux comprendre ses cheminements et ses évolutions dans le temps.

17 Dans un mode plus expérimental, nous développons une plateforme collaborative, OscarH (Osons les sciences dans la culture, les arts et l'histoire). Conçue avec l'aide de l'agence de 
conseil et de service Deuxième Labo et développée par une entreprise de développement web locale (Actency), cette plateforme collaborative est un site web modulable et évolutif, d'une nouvelle génération, qui s'inscrit dans une démarche de "Recherche et Développement" appliquée en matière de transmission et de circulation de l'information avec l'apparition des premiers outils de web sémantique. Elle fait également écho aux projets d'archives ouvertes mis en place dans les universités depuis quelques années et en cours de construction à Strasbourg. Il s'agit de développer à la fois une base d'informations détaillées sur le patrimoine universitaire, les collections et leur histoire, mais également une véritable plateforme sociale d'engagement s'appuyant sur la collaboration entre des acteurs d'horizons variés. L'objectif est d'offrir une pluralité de regards sur le patrimoine de l'université et favoriser l'engagement d'un dialogue sur des questions science et société en les replaçant dans une perspective historique. Cette plateforme sera ouverte à l'automne 2016.

\section{Renforcer le dialogue science et société : inventer un nouveau musée}

18 Déployé par le ministère de l'Enseignement supérieur et de la Recherche en 2010 , l'Opération Campus, soutient la rénovation et le développement immobilier de plusieurs universités françaises. L'université de Strasbourg figure parmi les lauréats et une vingtaine de projets doivent recevoir des financements de ce programme. Parmi les projets retenus pour Strasbourg figure le Pôle culture science et société porté par le Jardin des sciences. Celui-ci se décline en deux opérations complémentaires : un nouveau bâtiment d'une part, comprenant un espace d'accueil commun à l'ensemble des structures muséales et un nouveau planétarium, construit entre l'Institut de zoologie et l'Institut de géologie; la rénovation du musée zoologique et la réhabilitation de l'ensemble de l'Institut de zoologie en structure muséale d'autre part. Ce nouveau pôle apparaît comme un tournant décisif pour le Jardin des sciences, le musée zoologique et les structures muséales de l'université pour accueillir un public nouveau et en plus grand nombre à l'horizon 2019 et réfléchir à la construction de nouveaux modes de mise en dialogue des sciences et de la société.

Il ne s'agit pas de construire un nouveau type de musée de science : le véritable challenge consiste à imaginer un nouveau musée dans lequel les sciences peuvent être replacées dans le contexte de leur production, leur usage technique, économique et politique. Parmi les nombreux défis figurent notre capacité à dépasser les enjeux institutionnels, ou encore à défier la traditionnelle organisation disciplinaire des musées et aussi (peut-être surtout) à offrir une discours alternatif et moins consensuel dans la sphère publique. Si l'on bénéficie d'un certains nombre d'expériences sur lesquelles s'appuyer, il y a encore de nombreuses questions auxquelles nous devons répondre, un mode opératoire à définir pour mobiliser les communautés universitaires, tant en sciences qu'en sciences humaines et sociales, mais également proposer de nouveaux vecteurs de dialogue, de rencontre et de participation des citoyens. 


\section{NOTES}

1. Charle, C. Jalons pour une histoire transnationale des universités, Cahiers d'histoire, $\mathrm{n}^{\circ} 121,2013$, pp. 21-42

2. Rapport IGA-ENR, La diffusion de la culture scientifique : bilan et perspectives, février 2012 ; Blandin, M.-C. et Magner, J.-B. La gouvernance de la culture scientifique, technique et industrielle, Rapport d'information fait au nom de la commission de la culture, de l'éducation et de la communication et du groupe d'études sur l'éducation populaire et la culture du Sénat, février 2013 ; Sauvageot C., et Stoeffler-Kern, F. Les filières scientifiques et l'emploi, Les Dossiers, $\mathrm{n}^{\circ} 177$, MESR, septembre 2006 ; Rapport Vincent Berger au Président de la République, 17 décembre 2012, assises de l'enseignement supérieur et de la recherche, paragraphe 73.

3. AMCSTI, EUSCEA, ECSITE, ICOM

\section{RÉSUMÉS}

Initiée dans La Lettre de l'OCIM en 2015 à l'occasion des 30 ans de l'OCIM, la rubrique "Quoi de neuf ?" propose un retour sur un article marquant de l'histoire de la revue. À partir d'un corpus d'articles sélectionnés par la rédaction de La Lettre de l'OCIM, les membres du comité des Publications de l'OCIM ont sélectionné plusieurs contributions. Dans cette perspective, il a été demandé à l'auteur ou à un expert du domaine de revisiter la problématique exposée dans l'article à la lueur des changements intervenus, notamment dans les pratiques professionnelles, depuis son écriture et de proposer des éléments prospectifs sur la question.

Sébastien Soubiran revient sur son article "Patrimoine des universités et médiation scientifique" publié en 2007 dans le $n^{\circ} 119$ de La Lettre de l'OCIM qui présentait la démarche de préservation du patrimoine scientifique de l'université Louis Pasteur de Strasbourg ainsi que les actions de médiation qui l'accompagnaient.

\section{INDEX}

Mots-clés : patrimoine, université, médiation scientifique

\section{AUTEUR}

\section{SÉBASTIEN SOUBIRAN}

directeur-adjoint du Jardin des sciences, université de Strasbourg

s.soubiran@unistra.fr 